\title{
ON THE MEAN MODULUS OF AN ANALYTIC FUNCTION
}

\author{
E. F. BECKENBACH, W. GUSTIN, AND H. SHNIAD
}

Throughout this paper $f=f(z)$ will denote an analytic function of the complex variable $z$ in the open unit circle $|z|<1$. The circle $C(r)$, on which $|z|=r$, of radius $r \geqq 0$ about the origin $z=0$ lies in the region of analyticity of $f$ provided $r<1$. For every positive real parameter $t(0<t<\infty)$ the mean of order $t$ of the modulus of $f$ on the circle $C(r)$ is defined as

$$
M_{t}(r ; f)=\left[\frac{1}{2 \pi} \int_{0}^{2 \pi}\left|f\left(r e^{i \theta}\right)\right| t d \theta\right]^{1 / t} .
$$

For fixed $f$ and $r$ this mean modulus $M_{t}(r ; f)$ as a function of $t$ is continuous, nonnegative, nondecreasing, and is bounded above by the maximum modulus of $f$ on $C(r)[1,2] .{ }^{1}$ Therefore the limit of $M_{t}(r ; f)$ exists as $t \rightarrow 0$ and $t \rightarrow \infty$. This limit is defined to be the mean modulus of $f$ on $C(r)$ of order 0 and of order $\infty$ respectively. It may be shown that the mean modulus of order 0 is the geometric mean of the modulus of $f$ on $C(r)$, which is simply evaluated by Jensen's formula, and that the mean modulus of order $\infty$ is the maximum modulus of $f$ on $C(r)[1,2]$. Thus $M_{t}(r ; f)$ is defined for all parameters $t$ in the compact infinite interval $0 \leqq t \leqq \infty$.

For fixed $f$ and $t(0 \leqq t \leqq \infty)$ the mean modulus $M_{t}(r ; f)$ as a function of $r$ in the interval $0 \leqq r<1$ is continuous, nonnegative, nondecreasing, and, except for the limiting parameters 0 and $\infty$, possesses a continuous derivative with respect to $r[1,3]$. Moreover, its logarithm is a nondecreasing convex function of $\log r$ (for $t=\infty$ this is the Hadamard three-circle theorem) $[1,3]$.

We shall be concerned here with the convexity of the mean modulus $M_{t}(r ; f)$ as a function of $r$. Let $T(f)$ be the set of all parameters $t$ in the compact infinite interval $0 \leqq t \leqq \infty$ such that $M_{t}(r ; f)$ is a convex function of $r$ in the interval $0 \leqq r<1$. Since $M_{t}(r ; f)$ is continuous with respect to the parameter $t$ and since any function which is the limit of convex functions is also convex, the set $T(f)$ is a closed and hence compact subset of the parameter interval $0 \leqq t \leqq \infty$. The set $T(f)$ need not, however, coincide with the entire parameter interval and indeed

Presented to the Society, December 30, 1947; received by the editors February 16, 1948.

${ }_{1}^{1}$ Numbers in brackets refer to the references cited at the end of the paper. 
may be a bounded subset of this interval as shown by the following example. Let

$$
f(z)=(z+\epsilon) /(1+\epsilon z), \quad|z|<1,0<\epsilon<1 .
$$

This linear fractional function maps the open unit circle onto itself and maps the circle $C(r)$ into a circle whose maximal distance from the origin is attained at the image point $f(r)$. Therefore

$$
M_{\infty}(r ; f)=f(r),
$$

which is easily seen to be a strictly concave function of $r$. Thus the set $T(f)$ for this function $f$ does not contain the parameter $\infty$ and hence, being closed, is bounded.

TheOREM. The set $T(f)$ contains the values $t=2 / k(k=1,2,3, \cdots)$ and their limit value $t=0$. Furthermore, if $f$ has at most $k$ zeros counting multiplicities $(k=1,2,3, \cdots)$, then $T(f)$ contains the closed interval $0 \leqq t \leqq 2 / k$.

Proof. Evidently the theorem is true for a constant function $f$, so we may assume that $f$ is nonconstant. We shall first investigate the convexity of the mean modulus $M=M_{t}(r ; f)$ in any open interval $\alpha<r<\beta$ such that the associated open annulus $\alpha<|2|<\beta$ contains no zeros of $f$.

Let the integer $m \geqq 0$ be the number of zeros, counting multiplicities, which lie in the closed circular interior, $|z| \leqq \alpha$, of the nonconstant function $f(z)$. If $m>0$, we denote these $m$ zeros, counting multiplicities, by $z_{h}(h=1, \cdots, m)$. The function

$$
A(z)=\prod_{1}^{m}\left(1-\frac{z_{h}}{z}\right)
$$

(where we understand any such product to have the value 1 if $m=0$ ) is then analytic and has no zeros in the open simply-connected circular exterior $|z|>\alpha$ including $z=\infty$. A single-valued function $a(z)$, analytic in this open circular exterior, then exists such that

$$
A(z)=a(z)^{2 / t} \text {. }
$$

Since $f(z)$ has no zeros in the open annulus $\alpha<|z|<\beta$, the only zeros of $f(z)$ in the open circular interior $|z|<\beta$ are the zeros $z_{h}(h=1, \cdots, m)$. Consequently the function

$$
B(z)=f(z) / \prod_{1}^{m}{ }_{h}\left(z-z_{h}\right)
$$

is analytic and has no zeros in the open simply-connected circular 
interior $|z|<\beta$. A single-valued function $b(z)$, analytic in this open circular interior, then exists such that

$$
B(z)=b(z)^{2 / t} \text {. }
$$

From (2), (3), (4), and (5) we see that

$$
f(z)=z^{m} A(z) B(z)=z^{m} a(z)^{2 / t} b(z)^{2 / t}=z^{m} c(z)^{2 / t}
$$

where the function $c(z)=a(z) b(z)$ is single-valued and analytic in the open annulus $\alpha<|z|<\beta$ and hence admits a Laurent expansion

$$
c(z)=\sum_{-\infty}^{\infty} c_{p} z^{p}
$$

in this annulus. In the associated interval $\alpha<r<\beta$ we define

$$
s(r)=\frac{1}{2 \pi} \int_{0}^{2 \pi}\left|c\left(r e^{i \theta}\right)\right|^{2} d \theta .
$$

This integral (8) may be evaluated in terms of the coefficients of the series (7) according to the well known formula

$$
s(r)=\sum_{-\infty}^{\infty}\left|c_{p}\right|^{2} r^{2 p}
$$

From (1), (6), and (8) we see that

$$
M=r^{m} s^{1 / t} .
$$

Differentiating this expression twice with respect to $r$ we obtain the formula

$$
\begin{aligned}
\mu & =t^{2} r^{2} s^{2} M^{\prime \prime} / M \\
& =t^{2} m(m-1) s^{2}+2 t m s r s^{\prime}+(1-t) r^{2} s^{2}+t s r^{2} s^{\prime \prime},
\end{aligned}
$$

where primes denote differentiation with respect to $r$.

Now consider the function

$$
N=r^{n} s^{1 / 2}
$$

where $n$ is an as yet undetermined integer. Differentiating this expression twice with respect to $r$ we obtain the formula

$$
\nu=4 r^{2} s^{2} N^{\prime \prime} / N=4 n(n-1) s^{2}+4 n s r s^{\prime}-r^{2} s^{\prime 2}+2 s r^{2} s^{\prime \prime} .
$$

Substitution of the power series (9) into (12) gives

$$
\nu=\sum_{-\infty}^{\infty}{ }_{p q} \nu_{p q}\left|c_{p} c_{q}\right|{ }^{2} r^{2 p+2 q}
$$


where the symmetrized coefficient $\nu_{p q}=\nu_{q p}$ has the value

$$
\nu_{p q}=(2 n+p+q-1)^{2}+3(p-q)^{2}-1 .
$$

By examining the two cases $p=q$ and $p \neq q$ it is easy to see, since $n, p, q$ are integers, that $\nu_{p q} \geqq 0$. Consequently $\nu \geqq 0$ and $N^{\prime \prime} \geqq 0$.

If $t=2$ and $n=m$, then $N=M$, whence $M^{\prime \prime} \geqq 0$. Therefore $M_{t}(r ; f)$ is a convex function of $r$ in $\alpha<r<\beta$ if $t=2$.

We now show that $M_{t}(r ; f)$ is a convex function of $r$ in $\alpha<r<\beta$ if $0<t<2$ and if the open interval

$$
I_{t}(m): t m-t<x<t m
$$

contains no even integer. Evidently the interval

$$
t m-t<x \leqq t m-t+2
$$

contains exactly one even integer $2 n$. Since $2 n$ does not lie in the interval $I_{t}(m)$ we conclude that

$$
t m \leqq 2 n \leqq t m-t+2,
$$

whence

$$
t m-2 n \leqq 0 \leqq t m-2 n+2-t .
$$

Now let this integer $n$ be the $n$ of (11). Consider the following expression quadratic in the variables $s$ and $r s^{\prime}: \phi=2 \mu-t \nu=2 t(t m(m-1)-2 n(n-1)) s^{2}+4 t(m-n) s r s^{\prime}+(2-t) r^{2} s^{\prime 2}$. The discriminant of this quadratic form is

$$
\Delta=8 t(t m-2 n)(t m-2 n+2-t) .
$$

Since $0<t<2$ the coefficient $2-t$ of $r^{2} s^{2}$ in $\phi$ is positive, and from (13) we see that $\Delta \leqq 0$. Therefore $\phi \geqq 0$ and, as we have already shown, $\nu \geqq 0$, so we conclude that

$$
2 \mu=\phi+t \nu \geqq 0,
$$

whence $M^{\prime \prime} \geqq 0$ in the interval $\alpha<r<\beta$.

The open interval $I_{t}(m)$ contains no even integer $2 h$, if $t$ is of the form $2 / k(k=2,3, \cdots)$; else we would have the inequality

$$
m-1<h k<m
$$

which is impossible since $h, k, m$ are integers. Moreover, it is evident that the open interval $\boldsymbol{I}_{t}(m)$ contains no even integer if $0<t<2$ and $m=0$ or if $0<t<2 / m$ and $m \geqq 1$. Therefore $M_{t}(r ; f)$ is a convex func- 
tion of $r$ in $\alpha<r<\beta$ if $t=2 / k(k=1,2,3, \cdots)$ or if $0<t \leqq 2 / k$ $(k=1,2,3, \cdots)$ and $m \leqq k$.

It is easy to see how the theorem follows from this result. As we have already mentioned, the mean modulus $M$ possesses a continuous derivative $M^{\prime}$ with respect to $r$ in the interval $0 \leqq r<1$ provided $0<t<\infty$. We have shown that under certain conditions $M$ is convex in any open subinterval of $0 \leqq r<1$ which contains no moduli of zeros of $f$; therefore under these conditions $M^{\prime}$ is nondecreasing in any subinterval closed in $0 \leqq r<1$ which contains no moduli of zeros of $f$ in its interior. Since the zeros of the nonconstant analytic function $f$ are isolated, the collection of all such closed subintervals covers the interval $0 \leqq r<1$. Thus $M^{\prime}$ is a nondecreasing function of $r$ and hence $M$ is a convex function of $r$ in this interval.

The theorem is proved except for inclusion of the value $t=0$. However, since $T(f)$ is closed and contains the values $t=2 / k$ $(k=1,2,3, \cdots)$ it also contains the limit value $t=0$. That $T(f)$ contains the value $t=0$ may also be seen directly from the Jensen formula for the geometric mean.

The following corollary concerns the length $l(r ; f)$ of the map under $f$ of the circle $C(r)$.

Corollary. Both the length $l(r ; f)$ of the map under $f$ of the circle $C(r)$ and the circular expansion ratio $l(r ; f) / 2 \pi r$ are nondecreasing convex functions of $r$ in the interval $0 \leqq r<1$.

Proof. It suffices merely to exhibit the following formula for the length of the map of $C(r)$ :

$$
l(r ; f)=\int_{0}^{2 \pi}\left|f^{\prime}\left(r e^{i \theta}\right)\right| r d \theta=2 \pi r \cdot M_{1}\left(r ; f^{\prime}\right),
$$

where $f^{\prime}$ is the derivative of $f$ and hence is analytic in the open unit circle.

THEOREM. If $f$ vanishes at the origin, then $T(f)$ contains the entire parameter interval $0 \leqq t \leqq \infty$.

Proof. The theorem is evidently true if $f$ is of the form $c z$ where $c$ is a constant. If $f$ is not of this simple form, then, since $f$ vanishes at $z=0$, there exists a nonconstant function $g$, analytic in the open unit circle, such that

$$
f(z)=z g(z) .
$$

For a fixed parameter $t$ in the interval $0<t<\infty$ let $F=M_{t}(r ; f)$ and $G=M_{t}(r ; g)$, so that 


$$
F=r G .
$$

We shall denote successive differentiations of a function with respect to $r$ by primes, and successive differentiations of the logarithm of a function with respect to $\log r$ by asterisks. In any interval $\alpha<r<\beta$ containing no moduli of zeros of the nonconstant analytic function $g$ the first and second prime and asterisk derivatives of $G$ exist and are connected by the following relations:

$$
\begin{aligned}
r G^{\prime} & =G G^{*}, \\
r^{2} G^{\prime \prime} & =G\left(G^{* *}+G^{* 2}-G^{*}\right) .
\end{aligned}
$$

Differentiating (14) twice with respect to $r$ and using these relations we obtain the formula

$$
r F^{\prime \prime}=r^{2} G^{\prime \prime}+2 r G^{\prime}=G\left(G^{* *}+G^{* 2}+G^{*}\right) .
$$

As we have already mentioned $G^{*} \geqq 0$ and $G^{* *} \geqq 0$, so that $F^{\prime \prime} \geqq 0$ in $\alpha<r<\beta$. The extension of convexity of $F$ to $0 \leqq r<1$ proceeds as before. Therefore $T(f)$ contains the interval $0<t<\infty$ and being closed also includes the limiting parameters $t=0$ and $t=\infty$.

According to Schwarz's lemma the maximum modulus on the circle $C(r)$ of an analytic function $f$, which maps the origin into itself and the open unit circle into itself, is not greater than $r$. The above theorem shows that this maximum modulus is also a convex function of $r$.

Although the mean modulus $M_{t}(r ; f)$ may not be convex in the entire interval $0 \leqq r<1$, it may be convex in some subinterval containing $r=0$. We define $\rho(t ; f)$ to be the length of the maximal such subinterval if one exists and to be 0 if no such subinterval exists. Since the limit function of convex functions is convex, it is clear that

$$
\rho(t ; f) \geqq \lim _{t^{\prime} \rightarrow t} \sup \rho\left(t^{\prime} ; f\right),
$$

whence $\rho(t ; f)$ is an upper semicontinuous function of $t$.

We now show that $\rho(t ; f)>0$ if $0 \leqq t<\infty$. We have already seen by example that $\rho(\infty ; f)$ may be 0 . Since $\rho(t ; f)=1$ if $t=0$, if $f$ is constant, or if $f$ vanishes at $z=0$, we may suppose that $0<t<\infty$ and that $f$ is a nonconstant analytic function which does not vanish at $z=0$. A neighborhood $|z|<\beta$ of $z=0$ then exists in which $f$ does not vanish. Thus, in the notation of our first theorem we have $m=0$ and $A(z)=1$. The expansion (7) is then a Taylor expansion with $c_{0} \neq 0$. Moreover, since $f$ is nonconstant, not all the coefficients $c_{1}, c_{2}, c_{3}, \cdots$ vanish. Let $c_{q} \neq 0$ be the first such nonvanishing coefficient. Substitution of the power series (9) into (10) gives 


$$
\mu=2 t q(2 q-1)\left|c_{\theta} c_{q}\right|^{2} r^{2 q}+O\left(r^{2 q+2}\right) .
$$

Since $q$ is a positive integer we infer that $\mu>0$ and hence $M^{\prime \prime}>0$ in some neighborhood of $r=0$. Consequently $\rho(t ; f)>0$.

We conclude with the following question: Is $\rho(t ; f)$ a continuous, non-increasing function of $t$ ?

\title{
REFERENCES
}

1. P6́lya and Szegö, Aufgaben und Lehrsätze aus der Analysis I, Berlin, 1925, pp. 143-144.

2. Hardy, Littlewood, Pólya, Inequalities, Cambridge, 1934, pp. 134-146.

3. Hardy, The mean value of the modulus of an analytic function, Proc. London Math. Soc. (2) vol. 14 (1915) pp. 269-277.

\author{
University of California, Los Angeles, \\ INDIANA UNIVERSITY, AND \\ University of California, Los ANgeles
}

